\title{
A Girl with Recurrent Brief Depressive Disorder and Cochlear Implantation; An Unusual Presentation
}

\author{
Reza Bidaki ${ }^{1,2}$, Seyyed Mojtaba Yassini ${ }^{1}$ and Mojgan Kian ${ }^{1 *}$ \\ ${ }^{1}$ Research Center of Addiction and Behavioral Sciences, Shahid Sadoughi University of Medical Sciences, Yazd, Iran \\ ${ }^{2}$ Yazd Diabetes Research Center, Shahid Sadoughi University of Medical Sciences, Yazd, Iran \\ ${ }^{*}$ Corresponding author: Mojgan Kian, Research Center of Addiction and Behavioral Sciences, Sha- \\ hid Sadoughi University of Medical Sciences, Yazd, Iran, Tel: +98 3532633555; Fax: +98 3532633555; \\ E-mail:Mo_kian2005@yahoo.com \\ Citation: Bidaki R, Yassini SM, Kian M (2016) A Girl with Recurrent Brief Depressive Disorder and \\ Cochlear Implantation; An Unusual Presentation. Gavin J Addict Res Ther 2016: 13-14.
}

Received: 22 May, 2016; Accepted: 10 June, 2016; Published: 24 June, 2016

\section{Introduction}

Recurrent Brief Depressive Disorder (RBDD) is a prevalent affective disorder with a similarly high risk of suicide behavior as Major Depressive Disorder (MDD) [1]. The diagnostic criteria for RBDD requires the presence of at least five out of nine depressive symptoms, as like as MDD, occurring at least once a month and last only a few days [1]. Previous studies showed that although the risk of major depression in childhood is relatively small, it substantially increases with adolescence [2]. A few studies demonstrated that children with hearing impairment show higher prevalence of depression than normal hearing children when reported by parents or caregivers or by children themselves $[3,4]$. In contrast, the one study that specifically examined the influence of cochlear implants on symptoms of depression found no differences between children with CI (Cochlear Implant) and a normal hearing control group [3].

In this case study we introduce a girl with hearing impairment, who has vertigo as the main symptom in context of RBDD.

\section{Case Report}

The patient is a 12-year-old girl who was referred to a psychiatric clinic by an (Ear, Nose, and Throat) ENT specialist. The patient complains of dizziness, loss of balance, decreased appetite, and social withdrawal, sensitivity to rejection, irritability, and separation anxiety. However, severe Sensory Neural Hearing Loss (SNHL) and history of left cochlear implant were observed at birth and from 6 years ago respectively.

The patient's symptoms have started since two years ago without mark and specific stressor and has worsened over the past 6 months. In every episode, the symptoms last for 2 days, and they have worsened after an intense physical activity, extreme fatigue, and being in crowded places. During this period, there has been a noticeable impairment in the child's social and academic activities.

\section{Paraclinic findings}

CBC: normal;

Liver function tests: normal;

Renal function tests: normal;

Thyroid function tests: normal;

Brain Computed tomography was normal. The ECG taken from the patient was normal with no positive finding. According to the patient's family history, the mother of the family had a history of depression and is taking antidepressants. However, the patient herself had no specific psychological and psychiatric problems. After two sessions of medical examination, psychiatric consultation, and review of the previous and new medical records, the patient was diagnosed with RBDD and immediately underwent treatment on an outpatient basis.

First, the patient was prescribed with Betahistine $8 \mathrm{mg}$ at night, Dimenhydrinate $50 \mathrm{mg}$ twice a day and Imipramine 25 $\mathrm{mg}$ at night. Two months after the first visit, the results were not satisfactory. Therefore, previous drugs were discontinued and the patient was prescribed with Lamotrigine and Citalopram $10 \mathrm{mg} /$ day. After the second visit, the recurrence rate of symptoms significantly decreased. In the next visit, the dose of citalopram increased to $20 \mathrm{mg}$. Finally, one month after the last dose modification, it was completely decreased, and only dizziness was reported by the patient after the discontinuation of citalopram for two days. The patient's 
morale has improved significantly, and according to the patient's family, her educational performance and ability to communicate with her family and other children have also improved.

The patient has been regularly receiving Citalopram $20 \mathrm{mg}$ daily and Lamotrigine $50 \mathrm{mg}$ daily for 6 months now since her next visits, and she has not reported any specific complication. The patient is also being completely monitored and treated in terms of the initial complaint and RBDD.

\section{Discussion}

Recurrent Brief Depressive Disorder (RBDD) is a subtype of mood disorders to which little attention has been paid so far. Patients with this disorder are either undiagnosed or diagnosed too late, and they usually do not respond well to treatment $[5,6]$. According to our search, the case reports on RBDD show that in most cases, the focus is on the ultimate treatment of such patients [7].

Whereas the case introduced in this paper is a little girl who was referred with completely physical manifestations. Despite repeated referrals to specialists, she was finally diagnosed with RBDD by a psychiatrist, and was fully recovered after undergoing treatment.

Therefore, this case report aims to introduce the patient and point out that some psychiatric disorders such as RBDD can begin with neurological symptoms and have clear manifestations of a psychiatric disorder after a while [8]. It is important to note that such patients should not be missed and they should be closely evaluated. Sometimes, some patients spend their time and money in clinics, while their disease could have been diagnosed and treated much faster by taking a careful history and paying attention to fine psychological and psychiatric points [9].

Our case hadn't criteria for bipolar and personality disorder. The episodes were intermittent and not related to the menstrual cycle. We suggest that all physicians notice to probable psychiatry sign and symptoms about every patient with neurologic symptoms like vertigo.

Moreover, this patient suffered from an organic disorder known as congenital hearing loss, which has led to her getting a cochlear implant. While the child was having consecutive dizziness, all the attention was given to ENT and neurological problems, which misled the medical team. About this patient, all accurate physical assessments were indicative of the lack of justification of internal, ENT, and neurological problems for the patient's dizziness. Although most chronic organic disorders increase the risk of psychiatric disorders such as anxiety and mood disorders in the long run, in this patient, the undiagnosed RBDD has been certainly the cause of specific problems such as dizziness [10].

Although this disease was previously considered to be a mood disorder spectrum, today, according to the Diagnostic and Statistical Manual of Mental Disorders Third Revision Fourth Edition (DSM-IV-TR), it is recognized as a completely independent disorder. Organic disorders, sleep disorder, alternating periods of depression, and high rate of suicidal tendency are observed among these patients. Therefore, it is very important to quickly identify and treat such patients $[1,5]$.

\section{Conclusion}

Recurrent brief depressive disorder can present as neurologic symptoms like vertigo and show a atypical presentation.

\section{References}

1. Pezawas L, Stamenkovic M, Kasper S (2001) Recurrent brief depressive episodes. Epidemiology, clinical aspects, diagnosis and therapy. Der Nervenarzt 72: $169-180$.

2. Chandler S, Howlin P, Simonoff E, O'Sullivan T, Tseng E, et al. (2015) Emotional and behavioural problems in young children with autism spectrum disorder. Dev Med Child Neurol 58: 202-228.

3. Sahli S, Arslan U, Belgin E (2009) Depressive emotioning in adolescents with cochlear implant and normal hearing. International Journal of Pediatric Otorhinolaryngology 73: 1774-1779.

4. Mance J, Edwards L (2012) Deafness-related self-perceptions and psychological well-being in deaf adolescents with cochlear implants. Cochlear Implants Int 13: 93-104.

5. Pezawas L (2010) Recurrent Brief Depressive Disorder. Encyclopedia of Psychopharmacology Springer 1142-1144.

6. Bartova L, Pezawas L (2015) Recurrent Brief Depressive Disorder. Encyclopedia of Psychopharmacology 1456-1459.

7. Angst J, Ajdacic-Gross V, Rössler W (2014) The clinical relevance and validity of brief Major Depressive Syndromes (MDS). Romanian Journal of Psychopharmacology 14: 1-8

8. Rich S, Levinger M, Werner S, Adelman C (2013) Being an adolescent with a cochlear implant in the world of hearing people: coping in school, in society and with self identity. Int J Pediatr Otorhinolaryngol 77: 1337-1344.

9. Moog JS, Geers AE, Gustus CH, Brenner CA (2011) Psychosocial adjustment in adolescents who have used cochlear implants since preschool. Ear Hear 32: 75-83.

10. Kramer T, Als L, Garralda ME (2015) Challenges to primary care in diagnosing and managing depression in children and young people. BMJ 350: 2512. 